\title{
CLUSTER observations of electron outflowing beams carrying downward currents above the polar cap by northward IMF
}

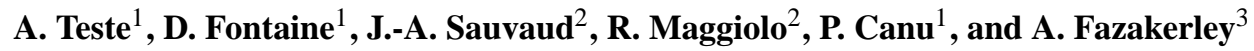 \\ ${ }^{1}$ CETP, 10-12 avenue de l'Europe, Vélizy 78140, France \\ ${ }^{2}$ CESR, 9 avenue du Colonel Roche, Toulouse 31028, France \\ ${ }^{3}$ MSSL, Holmbury St Mary, Dorking RH5 6NT, UK
}

Received: 22 December 2006 - Revised: 23 March 2007 - Accepted: 30 March 2007 - Published: 8 May 2007

\begin{abstract}
Above the polar cap, at about 5-9 Earth radii $\left(R_{E}\right)$ altitude, the PEACE experiment onboard CLUSTER detected, for the first time, electron beams outflowing from the ionosphere with large and variable energy fluxes, well collimated along the magnetic field lines. All these events occurred during periods of northward or weak interplanetary magnetic field (IMF).

These outflowing beams were generally detected below $100 \mathrm{eV}$ and typically between 40 and $70 \mathrm{eV}$, just above the photoelectron level. Their energy gain can be explained by the presence of a field-aligned potential drop below the spacecraft, as in the auroral zone. The careful analysis of the beams distribution function indicates that they were not only accelerated but also heated. The parallel heating is estimated to about 2 to $20 \mathrm{eV}$ and it globally tends to increase with the acceleration energy. Moreover, WHISPER observed broadband electrostatic emissions around a few $\mathrm{kHz}$ correlated with the outflowing electron beams, which suggests beam-plasma interactions capable of triggering plasma instabilities.

In presence of simultaneous very weak ion fluxes, the outflowing electron beams are the main carriers of downward field-aligned currents estimated to about $10 \mathrm{nA} / \mathrm{m}^{2}$. These electron beams are actually not isolated but surrounded by wider structures of ion outflows. All along its polar cap crossings, Cluster observed successive electron and ion outflows. This implies that the polar ionosphere represents a significant source of cold plasma for the magnetosphere during northward or weak IMF conditions. The successive ion and electron outflows finally result in a filamented current system of opposite polarities which connects the polar ionosphere to distant regions of the magnetosphere.
\end{abstract}

Keywords. Magnetospheric physics (Current systems; Magnetosphere-ionosphere interactions; Polar cap phenomena)

Correspondence to: A. Teste

(ate@cetp.ipsl.fr)

\section{Introduction}

To our knowledge, there is no report on upflowing electron beams observed above the polar cap. The main phenomenon concerning the electrons at polar latitudes is the precipitation into the ionosphere of accelerated electrons, known as the polar rain or the polar cap arcs: see for example the review by Zhu et al. (1997) or the statistical study by Shinohara et al. (1996).

The polar cap is better known as a region of ion outflows: polar wind, cleft ion fountain, outflowing ion beams, ... One important source is the ion upwelling from the cleft ion fountain: these ions are then detected above the polar cap while they undergo the anti-sunward convection (see the review by André and Yau, 1997; Yau and André, 1997). Locally accelerated ions also escape along polar magnetic field lines in the region corresponding to the polar arcs (Shelley et al., 1982). Recently, Maggiolo et al. (2006) identified both types of ion outflows from CLUSTER observations at high-altitudes, 5$9 R_{E}$ above the polar cap.

The situation is quite different for the auroral zone where the presence of anti-earthward, field-aligned electron beams has been reported from various observations, mainly at low altitude (Johnstone and Winningham, 1982; Klumpar and Heikkila, 1982; Burch et al., 1983; Marklund et al., 1994; Boehm et al., 1995; Carlson et al., 1998b). They appear highly collimated along magnetic field lines and generally have typical energies between a few tens and a few hundreds of $\mathrm{eV}$ with the more energetic events reaching the $\mathrm{keV}$ range (Cattell et al., 2004). The perpendicular temperature of these upgoing electron beams has been estimated as low as 0.2 $1 \mathrm{eV}$, comparable to ionospheric temperatures (Boehm et al., 1995; Carlson et al., 1998b). The auroral electron outflows can be observed in various situations: isolated, as part of bidirectional beams, or accompanied by ion conics events or ion precipitation (Elphic et al., 1998; Miyake et al., 1998; Carlson et al., 1998b). Their occurrence does not seem to be correlated with the IMF direction.

Published by Copernicus GmbH on behalf of the European Geosciences Union. 
Evans (1974) showed that the presence of parallel potential drops along magnetic field lines can be identified on the auroral electron energy spectra. Then, various studies revealed that the upflowing electron beams in the auroral region are associated with diverging electric fields, with an integrated potential that matches the beam energy (Burch et al., 1979; Marklund et al., 1994; Carlson et al., 1998a, b; Ergun et al., 1998; Mozer and Kletzing, 1998). It is admitted that the acceleration region is located above the ionosphere and below 1 or 2 Earth radii, or much lower depending on the observations, and that its thickness can extend over thousands of $\mathrm{km}$. The question is not closed since a more complex description recently emerged; it involves the presence of very large electric fields confined into very thin layers of the order of several Debye lengths (Andersson et al., 2002; Ergun et al., 2003).

Finally, these auroral upflowing electron beams have been identified as the main carriers of downward Birkeland currents in the dayside and nightside auroral region (McFadden et al., 1999). They contribute to auroral current densities of the order of a few $\mu \mathrm{A} / \mathrm{m}^{2}$ at altitudes inferior to 1 Earth's radius (Iijima and Potemra, 1976; Burch et al., 1983; Elphic et al., 1998).

In this paper, we present Cluster observations of outflowing electron beams detected above the polar cap at 5-9 $R_{E}$ altitude - higher than most auroral observations - during periods of northward IMF (Sect. 2). We estimate their main characteristics in Sect. 3 and we study their interaction with the ambient plasma and the resulting plasma instabilities in Sect. 4. With the background of auroral observations, we discuss the acceleration processes at their origin, in Sect. 5. In Sect. 6, we give an estimate of the global current system above the polar cap.

\section{Observations}

The four spacecraft of the Cluster mission were launched in 2000 on a quasi-polar orbit with an apogee at about 19 Earth radii $\left(R_{E}\right)$. They cross the polar cap at about 5 to $9 R_{E}$ altitude.

We mainly use data from Plasma Electron And Current Experiment (PEACE), which detects the electron fluxes between 0 and $26 \mathrm{keV}$ with 2 sensors located on opposite sides onboard each spacecraft: the High Energy Electron Analyser (HEEA) and the Low Energy Electron Analyser (LEEA), respectively dedicated to the higher and lower energy ranges. The field-of-view of each analyser covers $180^{\circ}$, divided into 12 polar zones, $15^{\circ}$ wide, relative to the spin axis. The azimuthal coverage of the 3-D distributions is achieved by spin rotation (Johnstone et al., 1997; Fazakerley et al., 2005). At altitudes of 5-9 $R_{E}$ over the polar cap, the plasma is generally tenuous and the spacecraft potential $\varphi_{\text {sat }}$ can reach as high values as $\sim 40$ Volts which accelerates the electrons. Consequently, the energy of the detected electrons is arti- ficially enhanced and it is necessary to subtract the energy corresponding to the spacecraft potential to recover the initial value (Szita et al., 2001).

We also use observations from other instruments onboard CLUSTER. The Cluster Ion Spectrometers (CIS) consist of two instruments: a Hot Ion Analyser (HIA) and a time-offlight ion COmpostion and DIstribution Function analyser (CODIF). They measure the ion distributions between $\sim 0$ and $40 \mathrm{keV/q}$ (Rème et al., 2001). The Electron Drift Instrument (EDI) permits to infer the electric field perpendicular to the spin axis (Paschmann et al., 2001). The Waves of HIgh frequency and Sounder for Probing the Electron density by Relaxation (WHISPER) experiment yields, in particular, the spectrum of natural plasma emissions in the frequency range 2-80 kHz (Décréau et al., 2001).

Typical observations during a northward IMF period above the polar cap are displayed in Fig. 1 which represents the ion and electron energy fluxes on 20 March 2003 for about half an hour, above the northern polar cap. The two bottom panels show that the electron fluxes are very weak in all directions - as illustrated by the bottom panel corresponding to the downgoing electrons along the magnetic field line except in one: the upward direction anti-parallel to the magnetic field (third panel). In this direction, PEACE detects the presence of successive outflowing electron beams, with quite large fluxes. They appear exactly between wider structures of ion outflow observed by CIS. As shown by the second panel, these ion outflows have typical inverted-V shaped energytime signatures. Their pitch angles of $180^{\circ}$ (top panel) confirm their motion in the upward direction along the magnetic field. Maggiolo et al. (2006) recently studied similar ion outflows above the polar cap and showed that their signature was consistent with the acceleration of ionospheric ions by a field-aligned potential drop. Thus, the polar cap ionosphere appears as a wide region of successive ion and electron outflows.

Generally, these upgoing electron beams are not isolated events. Many discrete structures are detected along the orbit over the whole polar cap during tens of minutes or even during hours, as illustrated by the 3 examples in Fig. 2. These events have similar characteristics: they are individually very narrow; usually detected during only a few spins $(\sim 30$ s to $1 \mathrm{~min}$ ). This gives a spatial width along the orbit of the order of $100-300 \mathrm{~km}$ at $\sim 5$ to $9 R_{E}$ altitude, corresponding to about $10 \mathrm{~km}$ at ionospheric altitudes, contrary to the wide surrounding ion outflow structures with spatial extents of the order of $\sim 1000 \mathrm{~km}$.

Most of the time, the maximum energy of the outflowing electron beams is well below $200-300 \mathrm{eV}$, as illustrated by the examples in Fig. 2. On 21 May 2002 (third panel), the average energy observed by the analyser LEEA is about $50 \mathrm{eV}$, just above the photoelectron strip below $40 \mathrm{eV}$ (in green). The spacecraft potential provided by the Electric Field and Wave experiment (EFW, Gustafsson et al., 2001) and displayed in the bottom panel of Fig. 2, reaches relatively high 

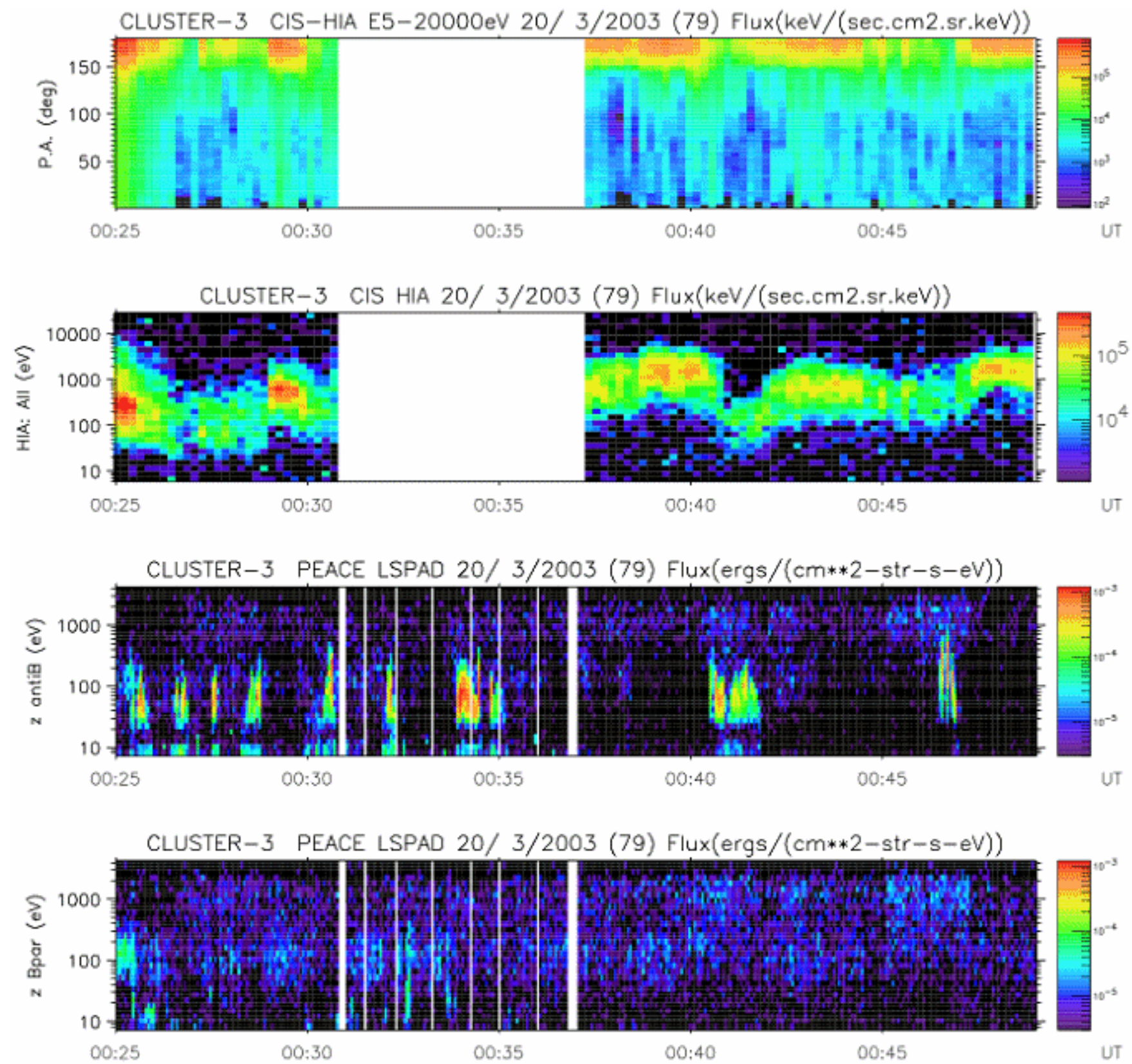

Fig. 1. First two panels: pitch angle and energy distributions of the ion energy fluxes observed by CLUSTER/CIS for 20 March 2003 , 00:25-00:50 UT. Last two panels: energy distribution of the electron energy fluxes observed by CLUSTER/PEACE for the same time period in the upward (antiB) and downward (Bpar) directions along the magnetic field lines.

amplitudes, $\sim 40 \mathrm{~V}$, quite typical above the polar cap. It decreases down to $15 \mathrm{~V}$ at 18:15 UT and before 18:30 UT in correlation with the presence of upward electron beams at lower energies. The amplitude of the spacecraft potential varies similarly to the lower energy limit of the beams. So, the detected electron beams are natural and not part of the photoelectrons. Over the polar cap, the energy range of the electron beams is generally embedded in the photoelectron range or rather just at the top of it. In these conditions, it is difficult to extract quantitative characteristics. In the following, we will concentrate on events with a beam energy range well separated from the photoelectron strip, i.e. either when the beams are more energetic or when the spacecraft potential is reduced due to the Active Spacecraft POtential Control (ASPOC) (Torkar et al., 2001).

The spatio-temporal aspects of the outflowing electron beams are illustrated in Fig. 3 which displays the outflowing electron fluxes detected on 15 October 2003 onboard 

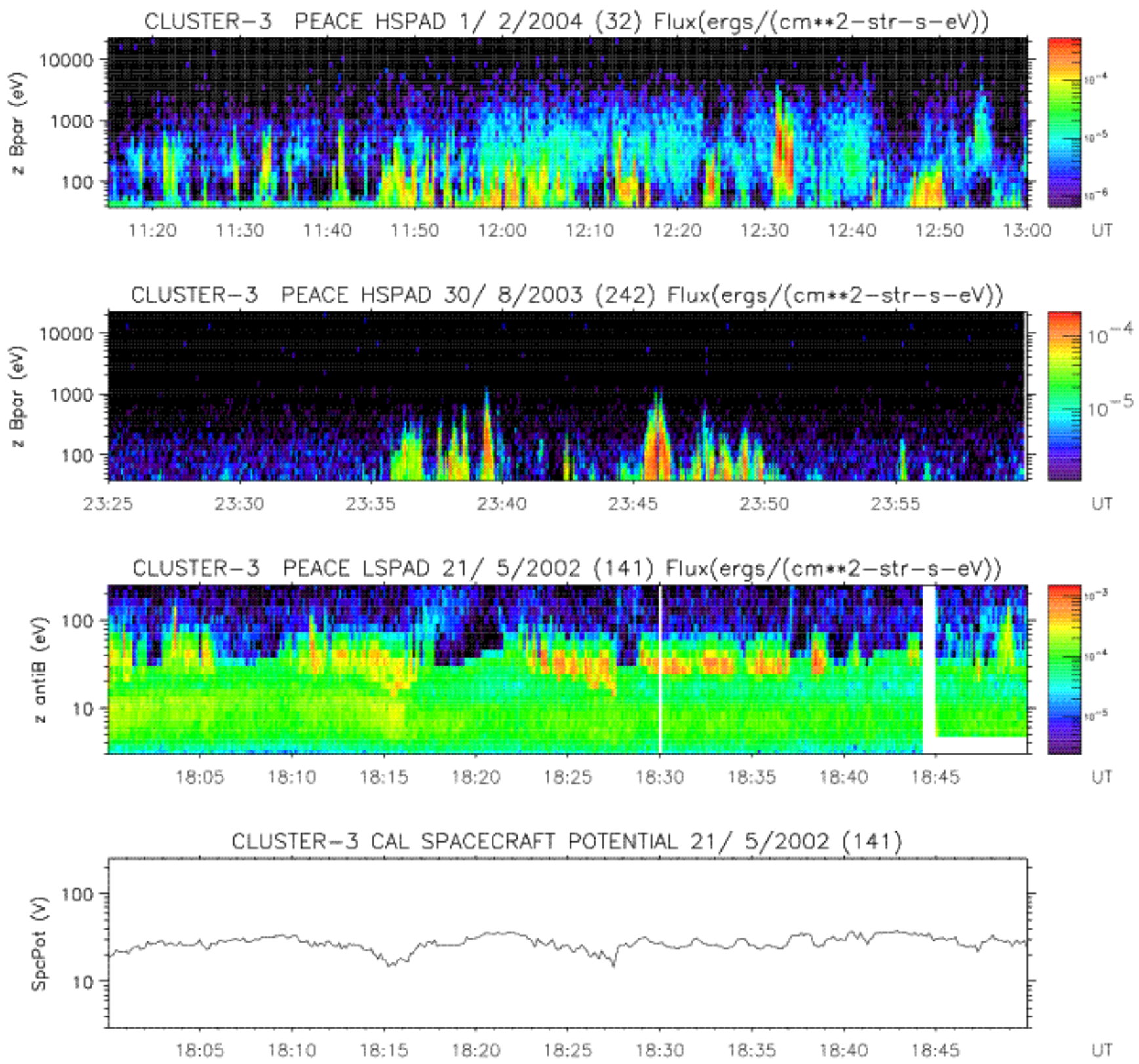

Fig. 2. Three top panels: electron energy fluxes in the upward direction along the magnetic field lines for three polar cap crossings: 1 February 2004 (top panel), 30 August 2003 (second panel) and 21 May 2002 (third panel). Bottom panel: spacecraft potential for 21 May 2002 in the same format as panel 3.

the 4 spacecraft, separated by $\sim 330$ to $720 \mathrm{~km}$. Between 01:26:10 UT and 01:30:40 UT, the whole fleet detects the same structure below $200 \mathrm{eV}$, but the fluxes differ from one spacecraft to the other one. This is particularly true for Cluster-1 and -2 , which are close to each other $(\sim 340 \mathrm{~km})$ and cross the structure approximately at the same time (less than one spin). This is also true for Cluster- 3 and -4 which also follow approximately the same orbit and cross the same structure with a very short time delay. Both comparisons show that the electron fluxes inside the outflowing beams vary very quickly. This is quite obvious in the case of the double beam detected by Cluster-3 between 01:38:00 and 01:41:30 UT. Cluster-1 and -2 detect it as two distinct structures while Cluster- 4 observes only one disturbed beam just in the time interval separating the two beams seen by Cluster1 and -2 . In summary, the fluxes of the outflowing electron beams vary at spatio-temporal scales shorter than the best resolution provided by the Cluster fleet; i.e., respectively, shorter than $350 \mathrm{~km}$ and one second. 

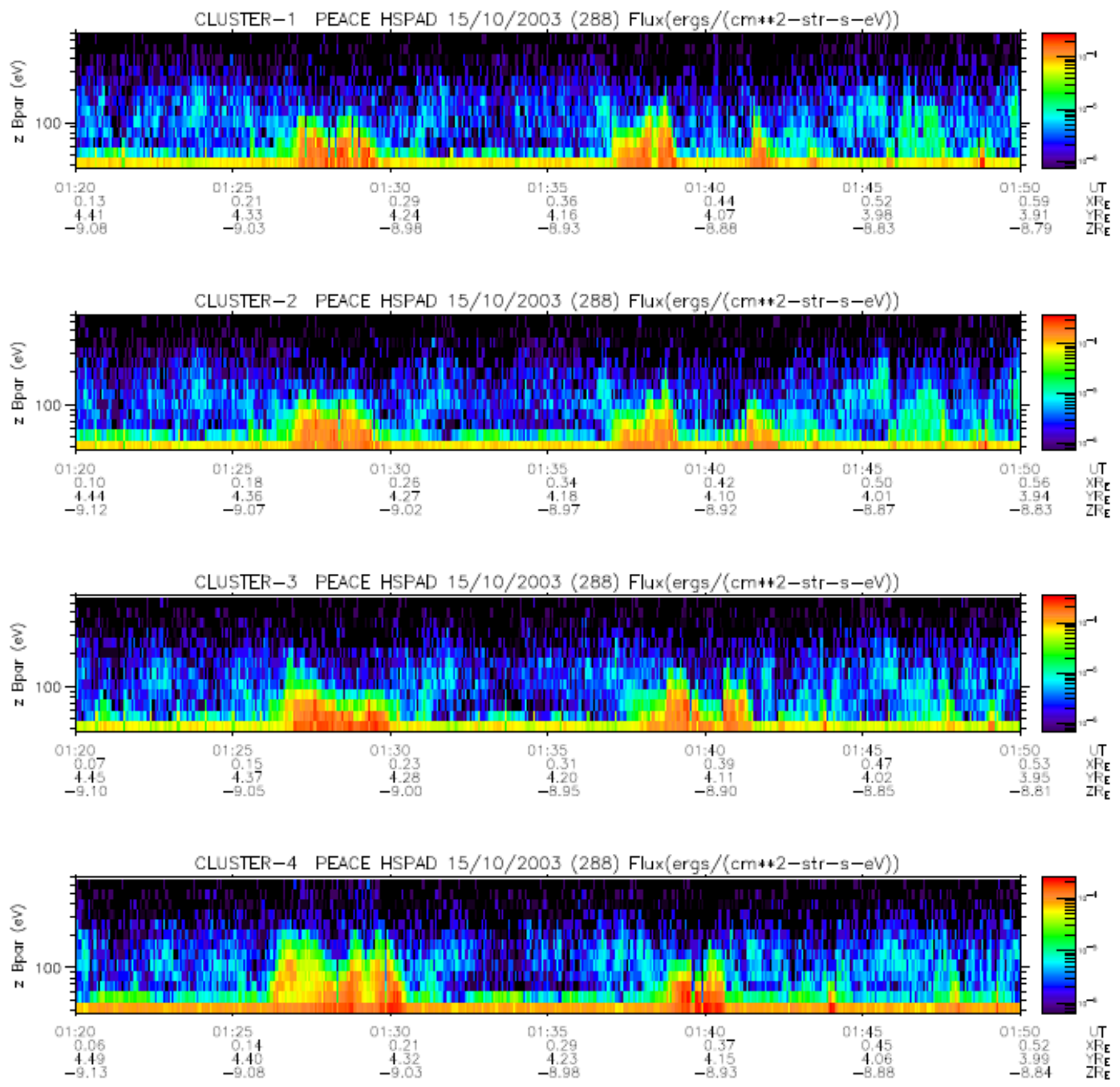

Fig. 3. Electron energy flux spectrograms in the upward direction along the magnetic field lines on 15 October 2003 between 01:20 and 01:50 UT, for the 4 spacecraft.

A more precise analysis of the PEACE data enables to more accurately describe the electron beams. Figure 4 displays examples of typical distribution functions observed during outflowing electron beams. On the right-hand side, the wheel representations give the pitch angle distribution function $f$ as a function of the energy (radial direction) between 10 and $1000 \mathrm{eV}$. The sensor field-of-view covers $180^{\circ}$. It is divided into twelve $15^{\circ}$-wide polar zones relative to the spin axis. The data have been re-organized relative to the magnetic field $\boldsymbol{B}$ (indicated by the red arrow). Only half of the wheel is actually measured and it has been duplicated on the second half which allows a better visualization of the field-aligned directions, but implicitly assumes a gyrotropic distribution. If we ignore the red circle at low energies due to photoelectrons, the distributions are almost depleted in all sectors except in the direction opposite to the magnetic field (sector $180^{\circ}$ ) where the highest values of $f$ are detected. In the Northern Hemisphere, they correspond to 

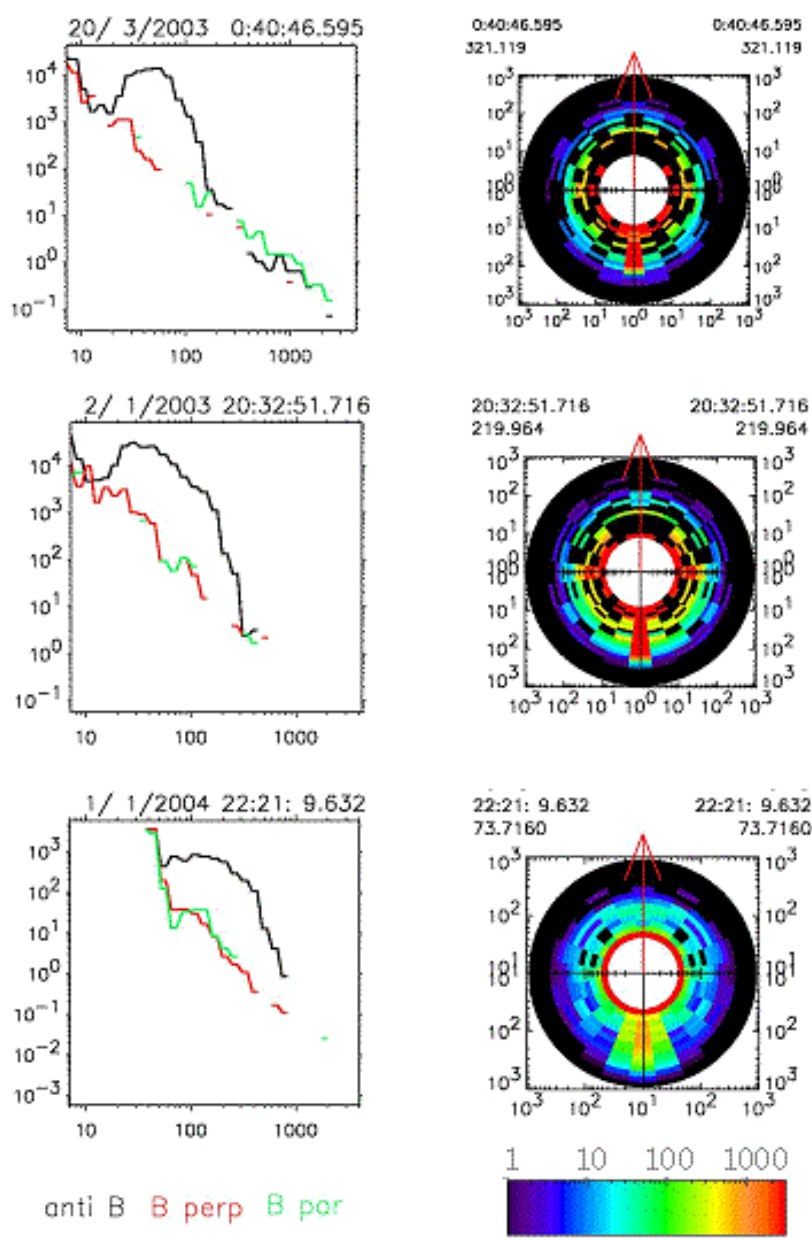

anti B B perp B por
In summary, field-aligned electron beams escaping from the ionosphere are observed along the polar cap magnetic field lines at altitudes between 5 and $9 R_{E}$. They occur mostly during periods of low geomagnetic activity with an IMF Bz component either weak or strongly northward ( $\sim 7 \mathrm{nT})$. Cluster observations reveal that these outflowing electron beams are not isolated events and that they are surrounded by wider structures of ion outflows: they follow one another along the spacecraft orbit, so that, during northward IMF conditions, the polar cap ionosphere appears as a wide region of outflowing particles, successively ions and electrons.

The structure of the outflowing electron beams differs significantly from the ion outflows in several aspects:

- they are much more narrow with typical spatial extents of the order of one hundred kilometres, i.e. one tenth of the surrounding ion outflow structures,

- the upward energy fluxes are very large and reach $10^{-4}$ $10^{-3} \mathrm{erg} /\left(\mathrm{cm}^{2}\right.$.sr.s.eV) at $\sim 5 R_{E}$; several orders of magnitude larger than the ion outflows in their vicinity. They correspond to about $10^{-2}-10^{-1} \mathrm{erg} /\left(\mathrm{cm}^{2}\right.$.sr.s.eV $)$ mapped at ionospheric altitudes and are comparable to auroral fluxes (Carlson et al., 1998b).

- inside the same structure, the fluxes are very variable at the scale of the best Cluster fleet resolution, around $350 \mathrm{~km}$ and one second.

- the maximum energy of their energy flux typically ranges between 30 and $200 \mathrm{eV}$. Some beams can even reach $1 \mathrm{keV}$, while others appear just at the top of the photoelectron strip at very low energy.

- they are collimated along the magnetic field direction, and their distribution function presents a well defined bump.

\section{Characteristics of the outflowing electron beams}

upgoing electrons. These beams are almost always observed within one angular sector, which means that they are actually well collimated along the magnetic field. This is comparable to what was observed in the auroral zone by Klumpar and Heikkila (1982), who reported on field-aligned upgoing ionospheric electron beams confined within $10^{\circ}$ of the magnetic field, at $1400 \mathrm{~km}$. The left-hand side of Fig. 4 shows a cut of the distribution function in 3 directions: parallel (green curve), perpendicular (red curve) and anti-parallel to $\boldsymbol{B}$ (black curve). After the largest values due to photoelectrons at low energies, the distribution function in the antiparallel direction (black curve) presents a very well-defined bump for the energies ranging from $20-40$ to $300-500 \mathrm{eV}$. This bump is the signature of an outward acceleration from the ionosphere up to the spacecraft altitude.
We now focus on the well-defined bump that the outflowing electron beams form in the distribution function to infer some of their characteristics. In order to do it, we first try to separate the beam contribution from the ambient plasma one. Of course, this requires that the electron beam energies are well separated from the photoelectron energies.

We cannot get any information on the distribution function in the direction perpendicular to the magnetic field because the beam only occurs in the angular sector containing the upward direction. Thus, in all other directions, the distribution function corresponds to the sole contribution of the ambient plasma $f_{p}$. In the upward direction, the total distribution function can be written as the sum of the contributions of the beam $f_{b}$ and of the ambient plasma $f_{p}$ : 

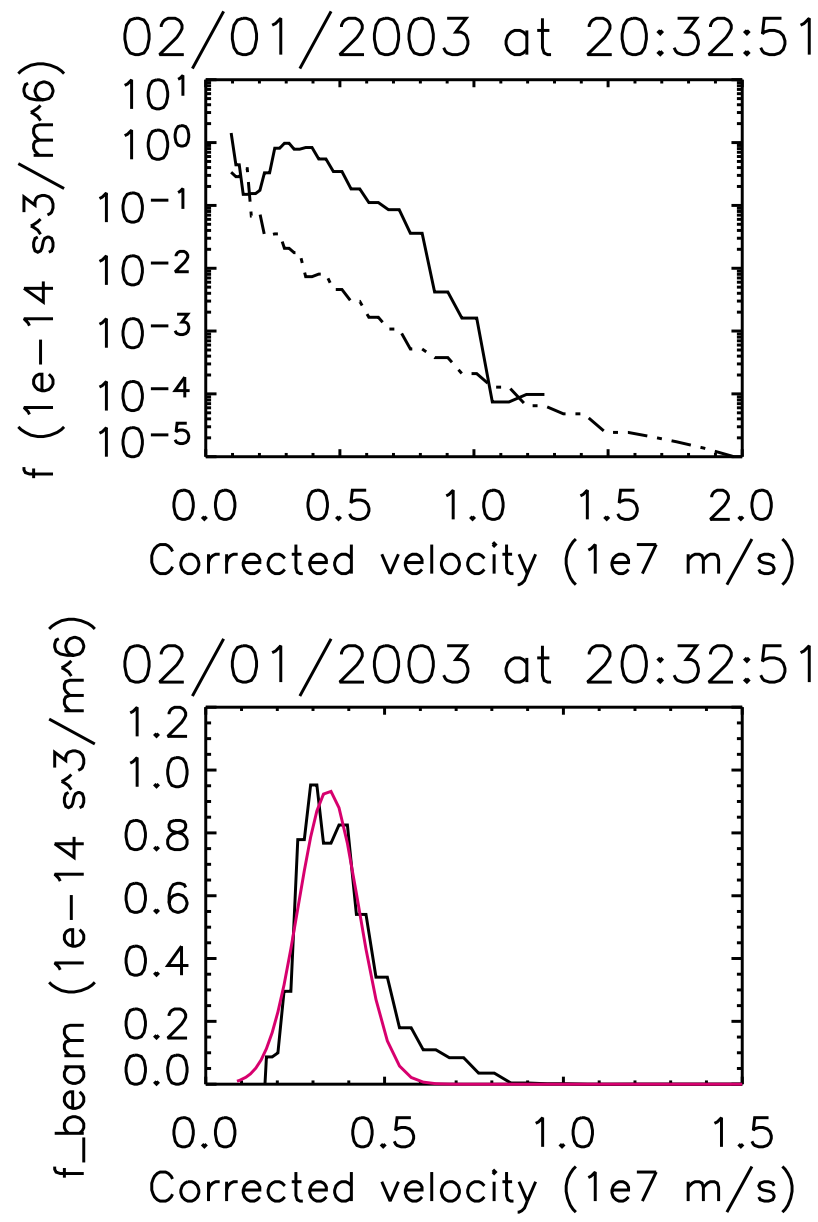

Fig. 5. Distribution function $f$ of 2 January 2003 at 20:32:51 UT as a function of the measured velocity corrected from the spacecraft potential. Top panel: upward component: $f_{/ / \text {tot }}$ (full curve), and the average of the 11 others: $f_{p}$ (dash-dotted curve). Bottom panel: distribution function $f_{/ / b}$ of the beam itself (black curve) and best Maxwellian fit (red curve).

$f_{/ / \text {tot }}=f_{/ / p}+f_{/ / b}$. The wheel representations of the pitch angle distribution functions in Fig. 4 show that the distribution functions are weak and almost isotropic everywhere out of the beam. An average over all the directions except the upward one provides a first estimate of the distribution function of the background plasma. The results are illustrated in Fig. 5 in the case of the electron outflow event observed at 20:32:51 UT on 2 January 2003. Top panel represents the total distribution function $f_{/ / \text {tot }}$ (full line) in the upward direction as a function of the velocity and an estimate of the ambient plasma contribution $f_{p}$ resulting from the average over the other directions (dash-dotted line). The difference between both provides an estimate of the beam contribution $f_{/ / b}$, which is illustrated in bottom panel (black curve) with a linear scale on both axes. It presents a peak around $3064 \mathrm{~km} / \mathrm{s}$ (i.e. $\sim 27 \mathrm{eV}$ ). The red curve represents the best fit by a Maxwellian distribution function with a mean par-

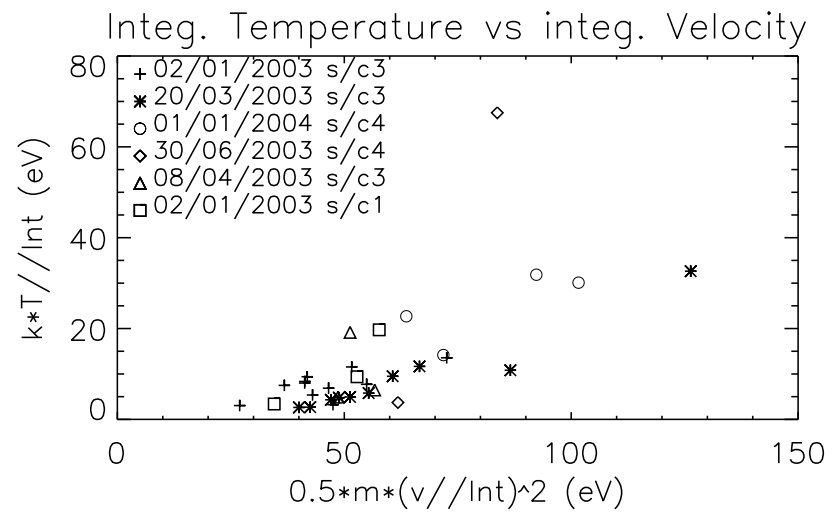

Fig. 6. Beam integrated temperature $T_{/ / \text {int }}$ as a function of the energy corresponding to their average velocities $\left(1 / 2 \mathrm{~m} . \mathrm{v}_{/ / \text {Int }}^{2}\right)$. The different symbols correspond to different days.

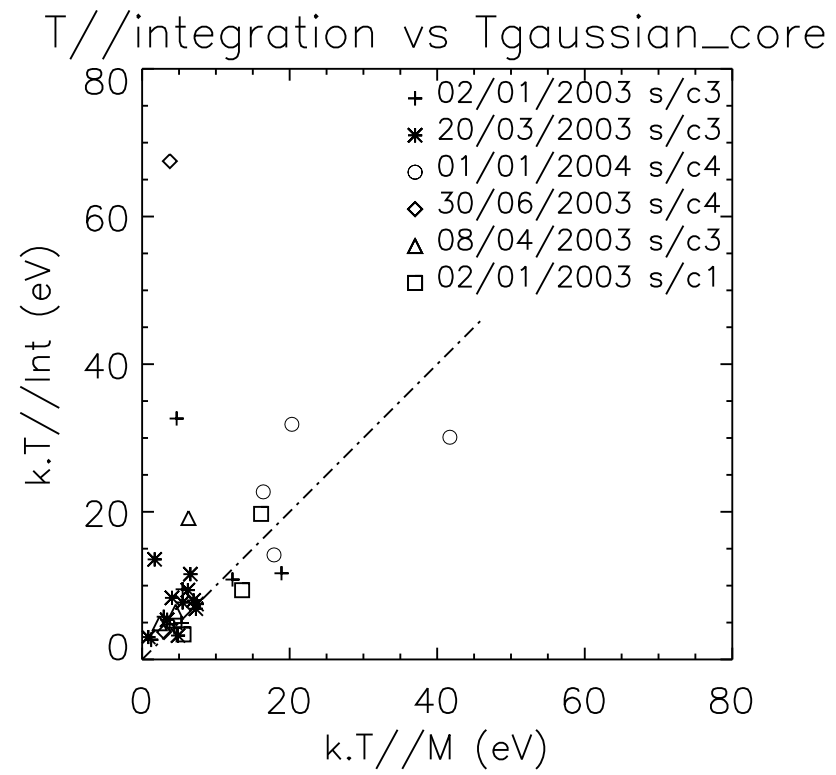

Fig. 7. Beam integrated temperature $T_{/ / \text {int }}$ as a function of the temperature $T_{/ / M}$ of the best Maxwellian fit. Same symbols as in Fig. 6.

allel drift velocity $v / / M 0(\sim 3429 \mathrm{~km} / \mathrm{s})$ corresponding to an energy of $33 \mathrm{eV}$ and a temperature of $4 \mathrm{eV}$. It reproduces satisfactorily the core of the observed distribution function and reveals the presence of a suprathermal tail at higher energies. It sometimes happens that a secondary peak exists near the maximum, as illustrated here at about $3832 \mathrm{~km} / \mathrm{s}(\sim 42 \mathrm{eV})$. An incorrect estimate of the beam contribution due to the calculation by subtraction cannot be excluded, but this secondary peak can also have a physical origin as the rapid variation of the acceleration processes during the measurements, thus leading to a variation of the beam velocities themselves. 
We then derive the beam drift velocity $v_{/ / \text {Int }}$ and the temperature $T_{/ / \text {Int }}$ by integration of the distribution function $f_{/ / b}$, as follows:

$$
\begin{aligned}
& n_{/ / \mathrm{Int}}=C^{t} \int_{-\infty}^{+\infty} f_{/ / b} d v_{/ /} \\
& v_{/ / \mathrm{Int}}=\frac{C^{t}}{n_{/ / \mathrm{Int}}} \int_{-\infty}^{+\infty} f_{/ / b} v_{/ /} d v_{/ /} \\
& \frac{1}{2} k T_{/ / \mathrm{Int}}=\frac{C^{t}}{n_{/ / \mathrm{Int}}} \int_{-\infty}^{+\infty} f_{/ / b} \frac{1}{2} m\left(v_{/ /}-v_{/ / \mathrm{Int}}\right)^{2} d v_{/ /}
\end{aligned}
$$

where $C^{t}$ is a constant and corresponds to the contribution of the perpendicular velocities.

The results are shown in Fig. 6 with the energy corresponding to the beam velocity $v_{/ / \mathrm{Int}}$ on the $\mathrm{x}$-axis and the beam temperature $T_{/ / \text {Int }}$ on the y-axis from the analysis of 33 distribution functions associated with 33 moments within 9 different events, marked with a specific symbol. Within a same day, several points correspond to different times of the same or different structures. There are no data below about $20 \mathrm{eV}$, because the beams are often close to the photoelectron energy range and it is difficult to extract reliable characteristics. The energy gained by the outflowing electron beams can reach $125 \mathrm{eV}$, but for most of them, it does not exceed $70 \mathrm{eV}$ with typical values around $40-70 \mathrm{eV}$. The beams are also heated to temperatures between $\sim 2$ and $\sim 20 \mathrm{eV}$, occasionally more. The results appear relatively dispersed, even inside a same event. Globally, a general trend emerges; the beam heating seems to increase with the energy gain.

The observed beam distribution functions often present a suprathermal tail. In order to determine the importance of this tail, we have fitted them by a Maxwellian distribution function $f_{/ / M}$ as follows:

$f_{/ / M} \propto \exp \left(-\frac{1}{2} \cdot \frac{m\left(v_{/ / M}-v_{/ / M 0}\right)^{2}}{k T_{/ / M}}\right)$

$v_{/ / M}, v_{/ / M 0}$ and $T_{/ / M}$ being, respectively, the parallel electron velocity, the mean parallel drift velocity and the parallel temperature of the Maxwellian.

The resulting Maxwellian temperature $T_{/ / M}$ is compared to the temperature estimated by integration $T_{/ / \text {Int }}$ in Fig. 7 . The symbols have the same meaning as in Fig. 6. The dash-dotted line represents purely Maxwellian beam distribution functions $\left(T_{/ / \text {Int }}=T_{/ / M}\right)$. Most of the 33 events analysed here are close to this line and thus well described by a Maxwellian. For some of them, located above the dashdotted line, the temperature $T_{/ / \text {Int }}$ estimated by integration is larger than the Maxwellian temperature; this reveals the importance of the suprathermal tail which contributes to enhance the total temperature above the Maxwellian core temperature. This is particularly true for temperatures above
$20 \mathrm{eV}$. Occasionally, some beams present an integrated temperature smaller than the Maxwellian fitted temperature. They correspond to double peak distributions which cannot be solved properly by a Maxwellian fit and would require further analysis to determine the eventual presence of a core or of multiple populations.

The analysis of the beam distribution function at successive times during the same event shows its variability (see the scattering of the points with the same symbol). Within one spin, a beam can evolve from a Maxwellian description to a very distorted distribution function with an important suprathermal tail.

In conclusion, we have analysed the characteristics of outflowing electron beams over the polar cap when they are detected at energies above the spacecraft potential, typically $20 \mathrm{eV}$. The core of their distribution function can be satisfactorily approached with a Maxwellian and they often present a more or less important suprathermal tail. From the ionosphere up to $5-9 R_{E}$ altitudes, they have gained an energy of the order of $40-70 \mathrm{eV}$, occasionally more. They have also been heated to temperatures typically between 2 and $20 \mathrm{eV}$, with the global trend that the heating increases with the energy gain. This heating suggests that these outflowing electrons have not only been accelerated but could also have experienced wave-particle interactions.

\section{Wave-particle interactions}

The wave experiments onboard Cluster do not show any signature of magnetic fluctuations during the events of electron outflows, at least for frequencies above $2 \mathrm{~Hz}$. Electric fluctuations are detected by the experiment Waves of HIgh frequency and Sounder for Probing the Electron density by Relaxation (WHISPER) in the passive mode (Décréau et al., 2001). Figure 8 shows broadband electrostatic emissions below about $6 \mathrm{kHz}$ around 22:58:30 UT on 2 January 2003 (bottom panel) strongly correlated with the electron beam observed at the same time which energy fluxes are maximum at $\sim 200 \mathrm{eV}$ (top panel). Such correlations are systematically observed for the other events.

Figure 9 shows the distribution function in the upward direction along the magnetic field observed at 22:58:32.633 UT during this event. The ASPOC experiment that controls the spacecraft potential and reduces the photoelectron fluxes was operating and gives access to the low energies. The contributions of the ambient plasma (red curve) and of the beam (black curve) are clearly separated. We assume that the ambient plasma can be described by a Maxwellian distribution function at rest (green curve). The best fit gives a plasma temperature of about $5 \mathrm{eV}$. Similarly, the beam can be fitted by a Maxwellian accelerated to an energy of $\sim 88 \mathrm{eV}$ and heated to a temperature of $\sim 4.4 \mathrm{eV}$ comparable to the plasma temperature. With a density of about $1.4 \times 10^{5} \mathrm{~m}^{-3}$, the ambient plasma appears much denser than the beam: 


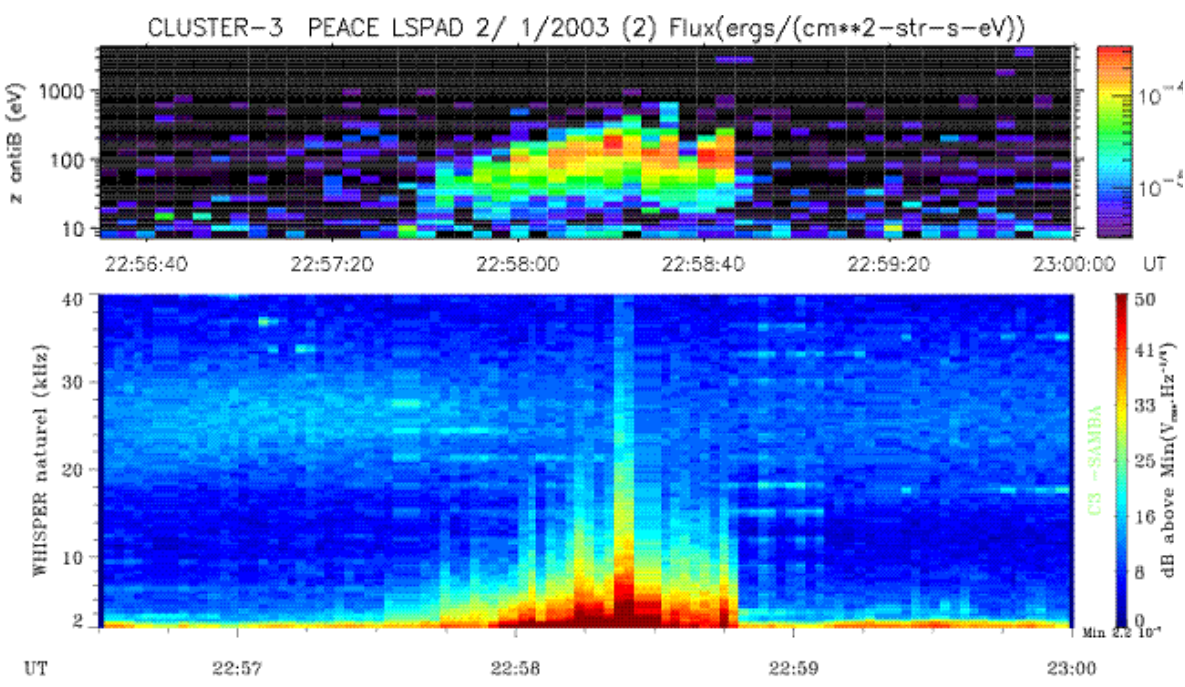

Fig. 8. Energy-time spectrogram of the upgoing electrons detected by PEACE (top panel) and electric field fluctuations observed by WHISPER (bottom panel) on 2 January 2003.

$\frac{n_{b}}{n_{p}} \sim 1.53 \%$, where $n_{b}$ and $n_{p}$ represent the beam and plasma densities. Similar conclusions can be drawn for the other cases: (i) the ambient plasma is much denser than the beam, with beam densities of the order of a few percents of the plasma density, (ii) the plasma temperature is comparable to the beam temperature which ranges essentially between 2 and $15 \mathrm{eV}$.

These values fulfil the conditions to trigger a resonant instability between a hot beam and a hot plasma: a beam density much smaller than the plasma one (Eq. 5) and a maximum growth rate of the kinetic instability superior to the Landau damping rate (Eq. 6) (Delcroix and Bers, 1994).

$n_{b}<<n_{p}$

$\frac{n_{b}}{n_{p}} \cdot\left(\frac{v_{0}}{v_{t h \_b}}\right)^{2}>\left(\frac{v_{0}}{v_{t h_{-} p}}\right)^{3} \cdot \exp \left(-\frac{v_{0}^{2}}{2 v_{t h_{-} p}^{2}}\right)$

where $v_{t h \_b}=\sqrt{\frac{2 \cdot k \cdot T_{b}}{m}}$ and $v_{t h_{-} p}=\sqrt{\frac{2 \cdot k \cdot T_{p}}{m}}$ stand for the beam and plasma thermal velocities. In our case, their respective values are $\sim 1.24 \times 10^{3} \mathrm{~km} / \mathrm{s}$ and $\sim 1.34 \times 10^{3} \mathrm{~km} / \mathrm{s}$, with the beam parallel drift velocity $\mathrm{v}_{0}$ equal to $\sim 5.58 \times 10^{3} \mathrm{~km} / \mathrm{s}$.

In this case, the oscillation real part $\omega_{r}$ at the maximum of the linear growth rate is estimated to (Delcroix and Bers, 1994):

$\omega_{r}^{2} \sim \omega_{p e}^{2}\left(1+3 k_{r}^{2} \lambda_{D e}^{2}\right)$

where $k_{r}$ is the wave vector real part and $\lambda_{D e}$ the Debye length.

The terms on the right hand side can be estimated from the beam and plasma characteristics. We obtain a real oscillation $\omega_{r}$ close to the plasma oscillation: $\omega_{r} \sim 1.24 \omega_{p e}$. With $f_{p e} \sim 3.3 \mathrm{kHz}$ and $f_{r} \sim 4.1 \mathrm{kHz}$, the instability frequency is in

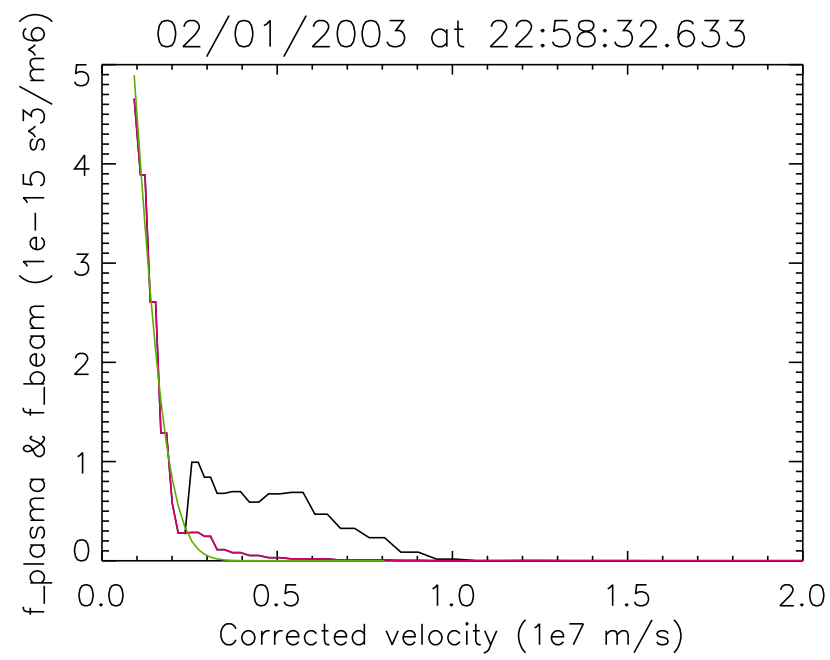

Fig. 9. Distribution functions of the beam $f_{/ / b}$ (black) and the ambient plasma $f_{p}$ (red) observed by PEACE on the 2 January 2003 at 22:58:32.633 UT with a Maxwellian fit of the plasma contribution (green).

good agreement with the frequency range $[\leq 2 \mathrm{kHz} ; \sim 6 \mathrm{kHz}]$ of the electrostatic emissions detected by WHISPER.

The other events behave similarly and this suggests that the upgoing electron beams observed by PEACE can likely trigger a hot beam - hot plasma instability and be responsible for the broadband electrostatic emissions detected by WHISPER. 

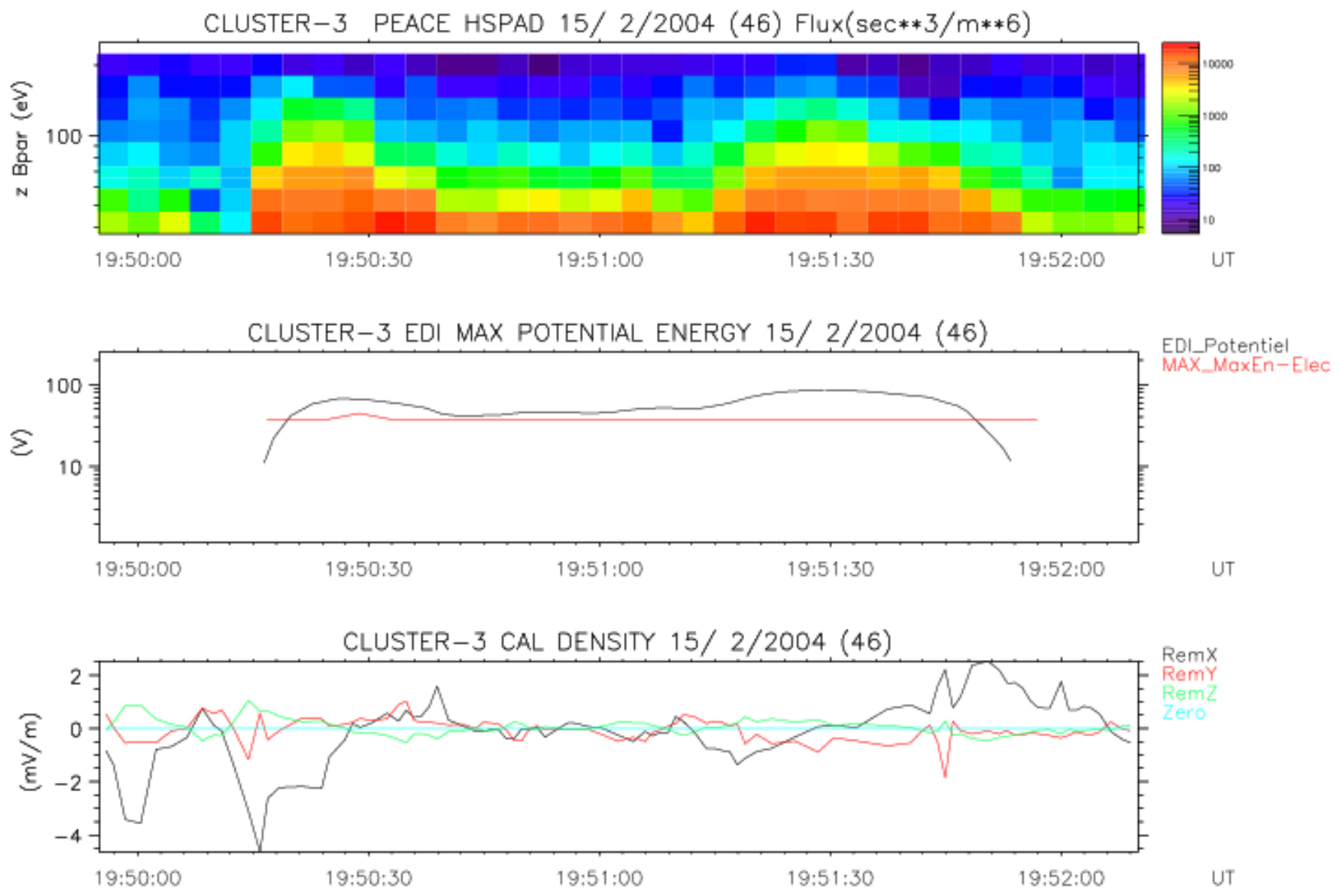

Fig. 10. Top panel: Distribution function spectrogram in the upward direction. Panel 2: Potential drops $\Phi_{\text {Emax }}$, computed from the energy of the distribution function maximum (red curve), and $\Phi_{\text {below_s/c }}$ from the electric field component perpendicular to the magnetic field (black curve). Last panel: components $\Delta E x$ (black), $\Delta E y$ (red) and $\Delta E z$ (green) of the electric field variations $\Delta \boldsymbol{E}_{\perp}$, in GSE coordinates.

\section{Electron acceleration source}

Observations of intense ionospheric upgoing electron beams have been reported at low altitude above the auroral zone (Klumpar and Heikkila, 1982; Marklund et al., 1994; Carlson et al., 1998a, b; Ergun et al., 2003). Their studies lead to the conclusion that they are accelerated by diverging electric fields in quasi-static potential structures. We test the same assumption in other conditions, above the polar cap and at higher altitudes.

The top panel of Fig. 10 displays the time variations of the distribution functions in the upward direction for an electron beam observed during almost 2 min on 15 February 2004, between 19:50 and 19:52 UT. The beam presents two bumps at both ends reaching $90 \mathrm{eV}$. The component of the electric field $\boldsymbol{E}_{\perp}$, perpendicular to the magnetic field $\boldsymbol{B}$, is estimated from the frozen field assumption: $\boldsymbol{E}_{\perp}=-\boldsymbol{V}_{d} \times \boldsymbol{B}$, with the magnetic field $\boldsymbol{B}$ measured by the Flux Gate Magnetometer (FGM) and the drift velocity $\boldsymbol{V}_{d}$ measured by the Electron Drift Instrument (EDI) along the spacecraft orbit (Balogh et al., 2001; Paschmann et al., 2001). This electric field component in- cludes the contribution of the large-scale convection electric field $\boldsymbol{E}_{\perp 0}$ which must be subtracted to calculate the fluctuations $\Delta \boldsymbol{E}_{\perp}$ associated with the electron beam.

$$
\Delta E_{\perp}=E_{\perp}-E_{\perp 0}
$$

The convection electric field is estimated by a polynomial fit to be of the order of $1 \mathrm{mV} / \mathrm{m}$. The 3 components of the resulting fluctuations $\Delta \boldsymbol{E}_{\perp}$ in the GSE coordinates system are displayed in the bottom panel of Fig. 10. They present a reversal, mainly in the $\mathrm{X}$ direction (black), essentially between $\pm 2.3 \mathrm{mV} / \mathrm{m}$ during the beam crossing. This suggests the presence of a diverging electrostatic shock below the spacecraft. In this case, the related parallel potential drop $\Phi_{\text {below s/c }}$ can be estimated from the integration of $\Delta \boldsymbol{E}_{\perp}$ along the spacecraft orbit $\boldsymbol{d l}$ (Mozer and Kletzing, 1998):

$\Phi_{\text {below s/c }}=\int_{\text {orbit }} \boldsymbol{\Delta} \boldsymbol{E}_{\perp} . \boldsymbol{d} \boldsymbol{l}$

It is represented by the black curve in the middle panel with 2 bumps reaching $\sim 70$ and $\sim 80$ Volts. Its shape approaches 
closely the beam energy variation $\Phi_{\text {Emax }}$ (red curve) determined from the maximum of the distribution function. The agreement between the electron energy gain and the estimated potential drop below the spacecraft supports the conclusion that an electron beam of ionospheric origin could have been accelerated by a potential drop below the spacecraft up to Cluster at $6.8 R_{E}$ altitude above the polar cap.

It must be outlined here that such a comparison between the electron energy gain and the potential drop computed from the electric field is generally quite difficult to handle from Cluster observations above the polar cap for several reasons due to:

- the spacecraft location. At several Earth radii above the polar cap, the plasma is very depleted and the photoelectrons reach quite high energy levels (several tens of $\mathrm{eV}$ ). When the outflowing electron beams come too close to this range, it becomes difficult to properly separate both contributions. Only energetic events with an energy range separated from the photoelectrons can be selected for further computations.

- the time resolution of PEACE data. Cluster spacecraft rotate at a 4-s spin rate. Except for the view direction aligned along the spin axis, any other direction is scanned once per spin, during $0.125 \mathrm{~s}$. This is generally the case for the magnetic field direction. In these conditions, the field-aligned beams are properly sampled if their time scale is much larger than $4 \mathrm{~s}$, while the electron outflow events, generally brief, typically last no more than a few spins. Their energy fluxes, often variable from one spin to the other one, indicate a shorter or at least comparable internal time-scale. The best candidates are events lasting at least several tens of seconds with relatively smooth flux variations.

- the simultaneous availability of electric field measurements.

Outflowing electron beams satisfying all these requirements, as the 15 February 2004 event studied above, are relatively exceptional. If they cannot give, on their own, a formal evidence that the polar cap electron outflows are accelerated by field-aligned potential drops, at least, they bring strong arguments in favour of it with the support of comparable studies at low altitudes above the auroral zone.

\section{Current density}

The following issue addresses the contribution of these intense outflowing electron fluxes to the polar cap current density.

The total field-aligned current density results from the contribution of all species:

$j_{/ /}=\sum_{s} j_{/ / s}$ with

$j_{/ / s}=n_{s} q_{s} v_{/ / s}$

where $q_{s}, n_{s}$ and $v_{/ / s}$ represent the electrical charge, density and field-aligned velocity of each species.

In all the analyzed events, the ion composition is largely dominated by the ions $\mathrm{H}^{+}$and the presence of the other ion species, including $\mathrm{O}^{+}$, is found quite negligible. In the following, the current contribution due to the ions $\mathrm{H}^{+}$will be simply noted $j_{/ / i}$. The $\mathrm{H}^{+}$densities $n_{i}$ and velocities $v_{/ / i}$, are derived from CIS measurements.

For the electrons, the moments are generally computed onboard from the integration of averaged 3-D distribution functions provided by PEACE over given energy windows (Fazakerley et al., 2005). For observations above the polar cap where the plasma is often very tenuous, the spacecraft potential increases and the photoelectron energy range may reach several tens of $\mathrm{eV}$. In such cases, the moments that take into account the lowest energy ranges may be polluted by the photoelectrons and the moments with higher energy thresholds may miss part of the low energy distributions involved in this study. An alternative method consists in using the 2D distribution functions $f_{e}$ available in the plane containing the magnetic field direction and the spin axis. At altitudes of a few Earth radii above the polar cap, the magnetic field is dominated by the steady and strong planetary contribution, which ensures a correct determination of the magnetic field direction onboard. Then, we assume the gyrotropy to derive the moments and, in particular, the electron current density $j_{/ / e-}$ :

$j_{/ / e-}=2 . \pi \cdot q_{e-} \cdot \int_{v} \int_{\theta=0^{\circ}}^{180^{\circ}} f_{e} \cdot v_{/ / e-} \cdot v^{2} \cdot \sin (\theta) \cdot d \theta \cdot d v$

The summation is actually computed over the 12 polar sectors describing the pitch angles $\theta$, between $0^{\circ}$ and $180^{\circ}$. As explained in Sect. 2, the magnetospheric electrons are accelerated by the spacecraft potential and the integration energy range should be shifted by the energy gain due to this potential to retrieve the energy range of the natural plasma.

The estimation and the comparison of the ion and electron contributions $j_{/ / i}$ and $j_{/ / e-}$ to the current density require intercalibrated quantities. In order to do it, we refer to the WHISPER experiment. The density ne_w can be computed from the electron plasma frequency $\omega_{p e}$ :

$\omega_{p e}=\sqrt{\frac{n_{e_{-} w} \cdot q_{e}^{2}}{\varepsilon_{0} \cdot m_{e}}}$

where $q_{e}$ and $m_{e}$ are the electron charge and mass and $\varepsilon_{0}$ the dielectric constant. The electron plasma frequency can be determined in the passive mode when a clear cut-off exists in the natural wave spectrum. Alternatively, the active mode excites plasma waves and the electron plasma frequency can 


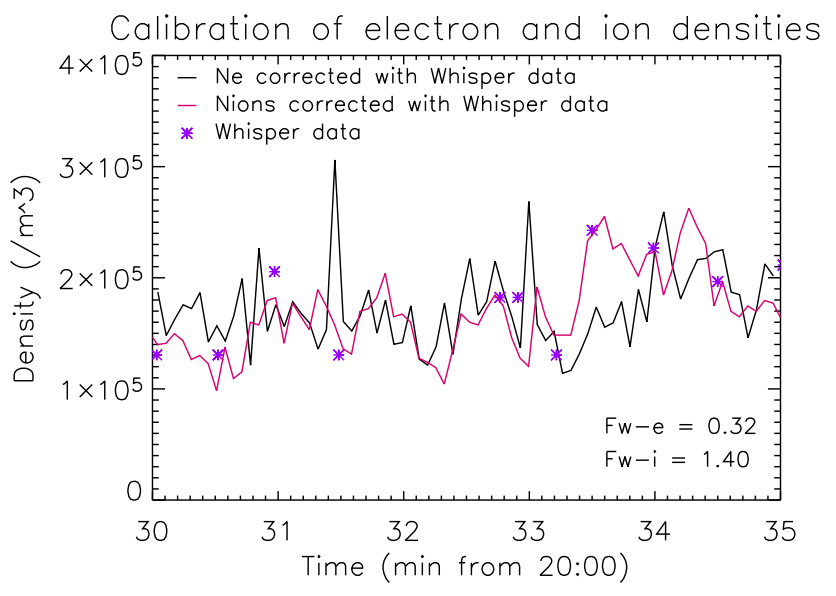

Fig. 11. Electron density from PEACE (black curve) and ion density from CIS (red curve) corrected with WHISPER data (stars).

be deduced from the upper hybrid and electron cyclotron frequencies if they signicantly differ (Décréau et al., 2001). Depending on plasma conditions and available data products, we apply one method or the other one. The resulting density ne_w inferred from WHISPER is then used to calibrate both the electron density, computed as the first moment of the distribution function $f_{e}$, and the ion density, assuming the quasi-neutrality.

An example of calibration is illustrated in Fig. 11 for the electron beam observed on 2 January 2003 after 20:30 UT. The stars represent the density ne_w inferred from WHISPER in the passive mode. They appear as relatively dispersed points because the identification of the electron plasma frequency is often difficult in regions of low density. To fit these values, we applied a multiplicative factor of about 0.32 to the electron density derived from PEACE (black curve) and $\sim 1.40$ to the ion density from CIS (red curve).

Finally, the computed field-aligned current densities are displayed in Fig. 12 for the events on 20 March 2003, already presented in Fig. 1. The plots in Fig. 12 concern the last part of the time period between 00:38 and 00:51 UT, after the ion data gap, with two intense outflowing electron beams surrounded by three wide structures of outflowing ions. The ion contribution (top panel) to the current density presents three wide bumps corresponding to the three ion structures. They carry upgoing (positive) currents with densities of the order of $1.5 \mathrm{nA} / \mathrm{m}^{2}$, occasionally reaching $2.3 \mathrm{nA} / \mathrm{m}^{2}$. Between the ion structures, the very small ion contribution is dominated by the electron contribution (middle panel) which carries much larger downgoing currents (negative). They reach $25 \mathrm{nA} / \mathrm{m}^{2}$ and $35 \mathrm{nA} / \mathrm{m}^{2}$, for the two outflowing beams, which correspond to 5 to $7 \mu \mathrm{A} / \mathrm{m}^{2}$ at ionospheric altitudes. Elsewhere, during the ion structures, the electron contribution fluctuates around zero. We obtained the ionospheric values from the magnetospheric ones by multiplying them by a factor 192 deduced from the Tsyganenko 87 magnetic field model (Tsyganenko, 1987).
The total current density resulting from both electron and ion contributions presents large fluctuations. To bring out the main trends, we averaged it over each structure. The results are displayed in the bottom panel of Fig. 12. The upgoing current densities (positive values), of the order of 0.7 to $1.4 \mathrm{nA} / \mathrm{m}^{2}$, are mainly carried by the outflowing ions. The downward current densities (negative values) reach much larger values, about 12.0 to $16.7 \mathrm{nA} / \mathrm{m}^{2}$, and are mainly transported by the outflowing electron beams. These results are quite typical of the studied events as shown by the average values $\left\langle j_{/ /}\right\rangle$reported in Table 1 . The calibration allowed us to estimate the error bar for $\left\langle j_{/ /}>\right.$of the order of $\pm 25 \%$ to $\pm 40 \%$. By northward IMF, successive current sheets of opposite polarities are detected along the spacecraft orbit over the polar cap. The upward currents of the order of $1 \mathrm{nA} / \mathrm{m}^{2}$ are mainly carried by outflowing ions while the upgoing electrons are the principal carriers of downward current densities of the order of $10 \mathrm{nA} / \mathrm{m}^{2}$, i.e., respectively, 0.2 and $2 \mu \mathrm{A} / \mathrm{m}^{2}$ at ionospheric altitudes. These values correspond to the order of magnitude of the current density found by Boehm et al. (1995), at $1700 \mathrm{~km}$ altitude in the auroral zone, from Freja data. They also correspond to the current density of $7 \mu \mathrm{A} / \mathrm{m}^{2}$ estimated by Araki et al. (1984) for the upflowing NBZ Birkeland currents (Iijima et al., 1984), in the polar cap, at 250 to $550 \mathrm{~km}$ altitude, from MAGSAT data.

From these observations, we derive and compare the total current carried by the successive current sheets. The current densities are aligned along the magnetic field lines. If $l_{\perp 1}$ and $l_{\perp 2}$ are the sheet dimensions in the plane perpendicular to $\boldsymbol{B}$, the current intensity $I$ is equal to:

$I=\iint j_{/ /} \cdot d l_{\perp 1} \cdot d l_{\perp 2}$

The current variation is observed along the spacecraft orbit s, i.e. along only one dimension: $d l_{\perp 1}=\cos \chi$. $d s$, where $\chi$ is the angle between the spacecraft orbit and the normal to the sheet. Without detailed information in the other direction, we aim at getting orders of magnitude by assuming that the current densities are homogeneous along $l_{\perp 2}$. The current intensity can then be expressed as:

$I=l_{\perp 2} \cos \chi \int j_{/ /} . d s$

where $l_{\perp 2}$ and $\cos \chi$ are unknown. If we assume, in a first approximation, that successive sheets have the same perpendicular extension $l_{\perp 2}$ and the same orientation $\chi$, the current that they carry is proportional to the integral $I_{\text {orbit }}$ :

$I_{\text {orbit }}=\int j_{/ /} \cdot d s$

where $I_{\text {orbit }}$ represents the current density per unit of the distance along the perpendicular direction $\perp_{2}$. The values of $I_{\text {orbit }}$ for successive sheets of opposite polarities are given in Table 1 . They are quite close for some cases, but depart by 
Table 1. Characteristic magnitudes of the upgoing particle acceleration structures for different events including the spatial extent of the structure along the satellite trajectory, the measured energy $E_{\text {meas }}$ of the beam distribution function maximum, the spacecraft potential, the current density $\left\langle j_{/ /}>\right.$averaged over the structure and the current intensity $I_{\text {orbit }}$.

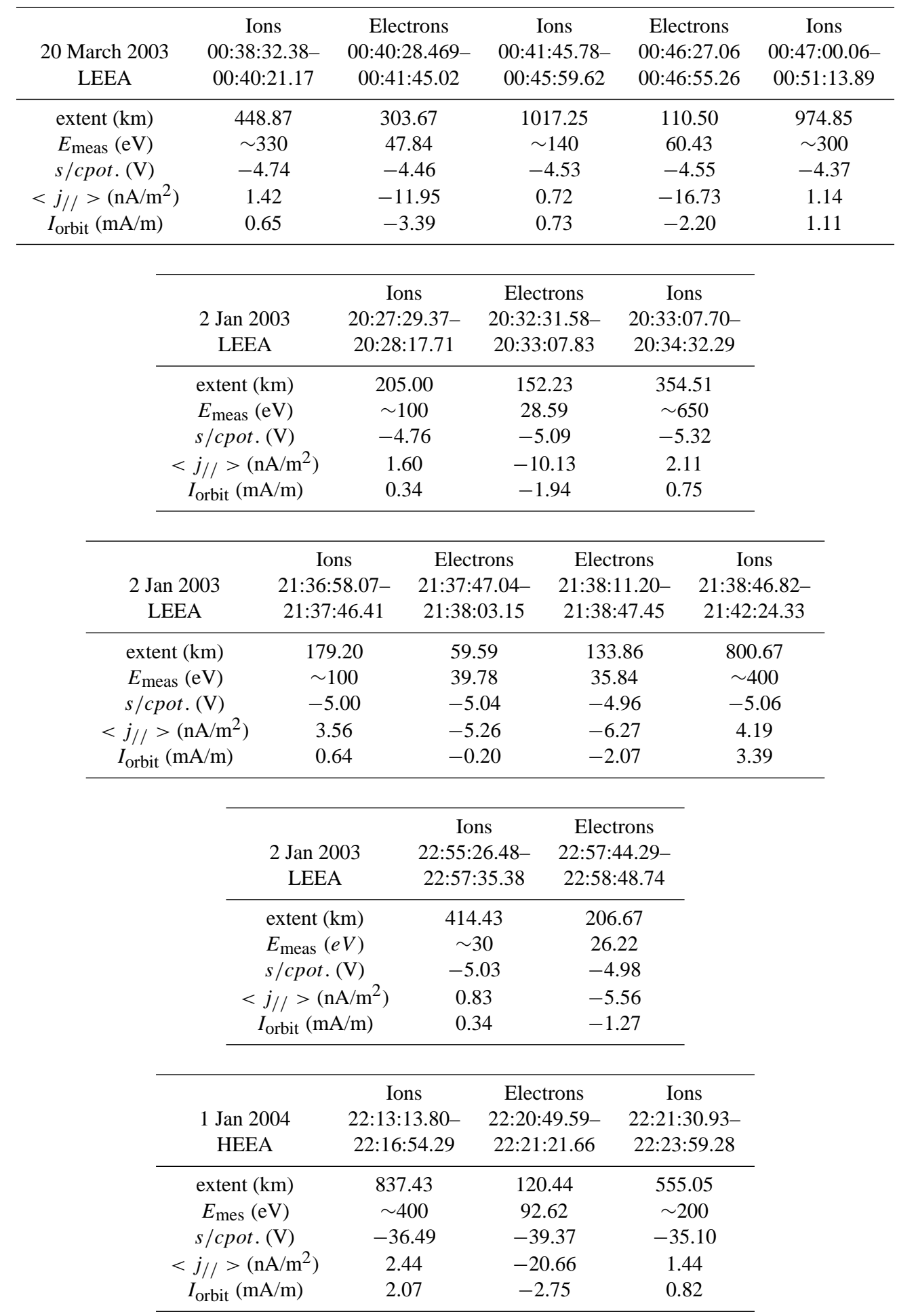



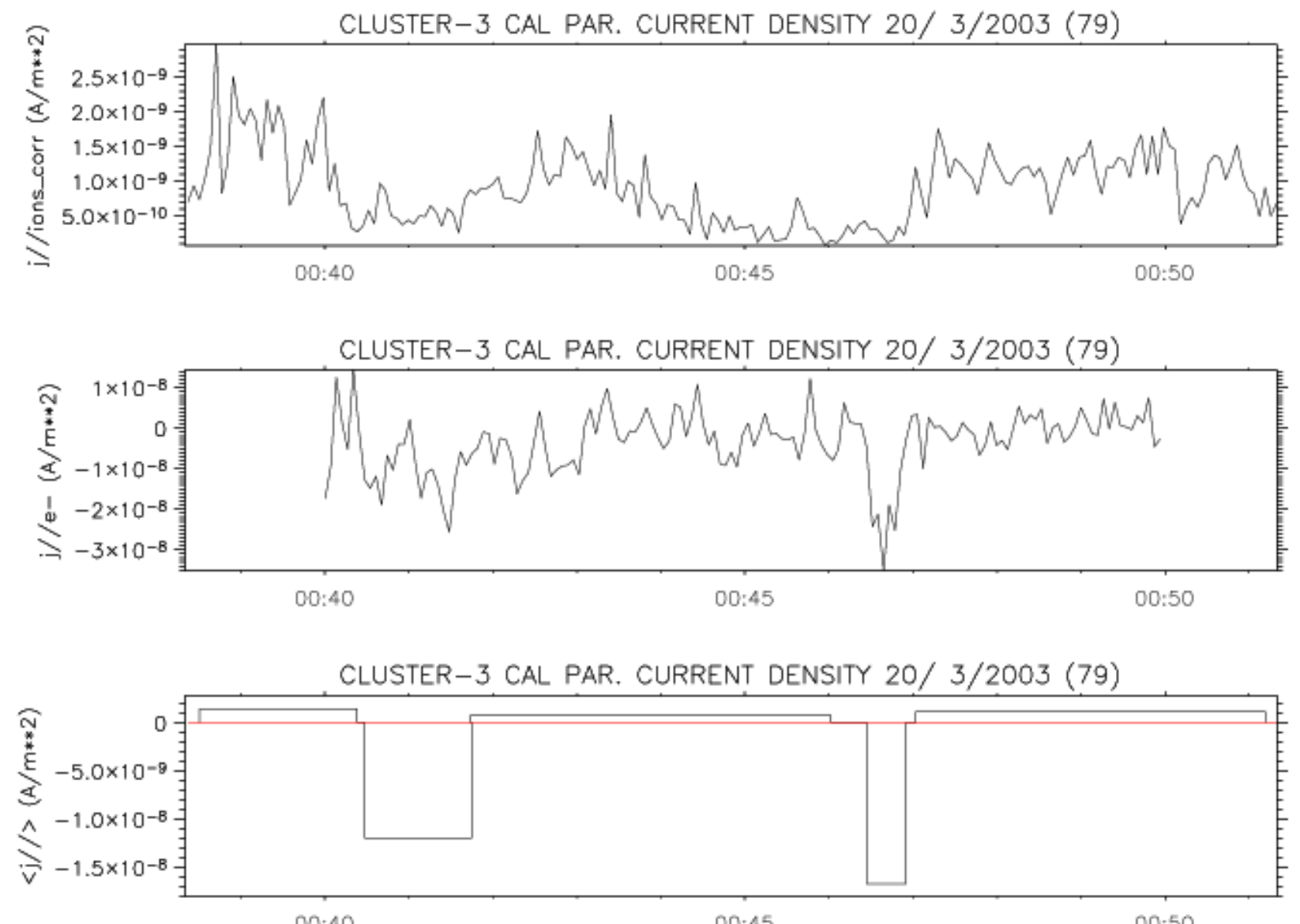

$00: 40$

$00: 45$

$00: 50$

Fig. 12. Component of the current density $j_{/ /}$along the magnetic field carried by the ions (top panel) and electrons (second panel), for 20 March 2003 between 00:38:20 and 00:51:20 UT and total parallel current density averaged over each structure (bottom panel).

a factor of about 2 or more for others. Indeed, it is difficult to discuss further the comparison because the moments derived from ion and electron measurements do not have neither the same time and space resolutions nor the same computation assumptions: 3-spin averages of the full distributions for the ions, gyrotropy assumption of 2-D distributions for the electrons. These differences come in addition of specific measurement geometries and uncertainties. Therefore, it is not expected to reach error bars better than a significant fraction of order of magnitude. Within these limits, the quantities $I_{\text {orbit }}$ derived for successive current sheets can be considered as comparable in a first approach and of the order of the $\mathrm{mA} / \mathrm{m}$. Finally, if successive current sheets have the same size $l_{\perp 2}$ and the same orientation $\chi$, they would carry comparable current intensities $I$, which satisfies the current closure condition.

In summary, for northward IMF conditions, Cluster observed, above the polar cap, successive current sheets of opposite polarities carrying comparable current intensities. The downward current densities, of $\sim 10 \mathrm{nA} / \mathrm{m}^{2}$, are mainly carried by the upgoing electrons and the upward current den- sities, of a few $\mathrm{nA} / \mathrm{m}^{2}$, by the ion outflows. The current densities discrepancies are compensated by the sizes of the sheets which vary in opposite sense. This can be visualized in the bottom panel of Fig. 12 where the area of each rectangle is proportional to the current intensity carried by the current sheet. The total area of the wide and low rectangles representing the upward current intensity is comparable to the area of the narrow and high rectangles for the downward current intensity.

\section{Conclusion}

Above the polar cap, at altitudes of 5-9 $R_{E}$, for northward or weak IMF, Cluster spacecraft detected structures of intense electron beams outflowing from the ionosphere, very collimated along magnetic field lines. These structures have a small spatial extent, of the order of $100 \mathrm{~km}$ at the satellite altitude, corresponding to a few $\mathrm{km}$ in the ionosphere. Their energy fluxes are quite large, they can reach $10^{-4} \mathrm{erg} /\left(\mathrm{cm}^{2}\right.$.sr.s.eV $)$, i.e. $\sim 10^{-2} \mathrm{erg} /\left(\mathrm{cm}^{2}\right.$.sr.s.eV) at 


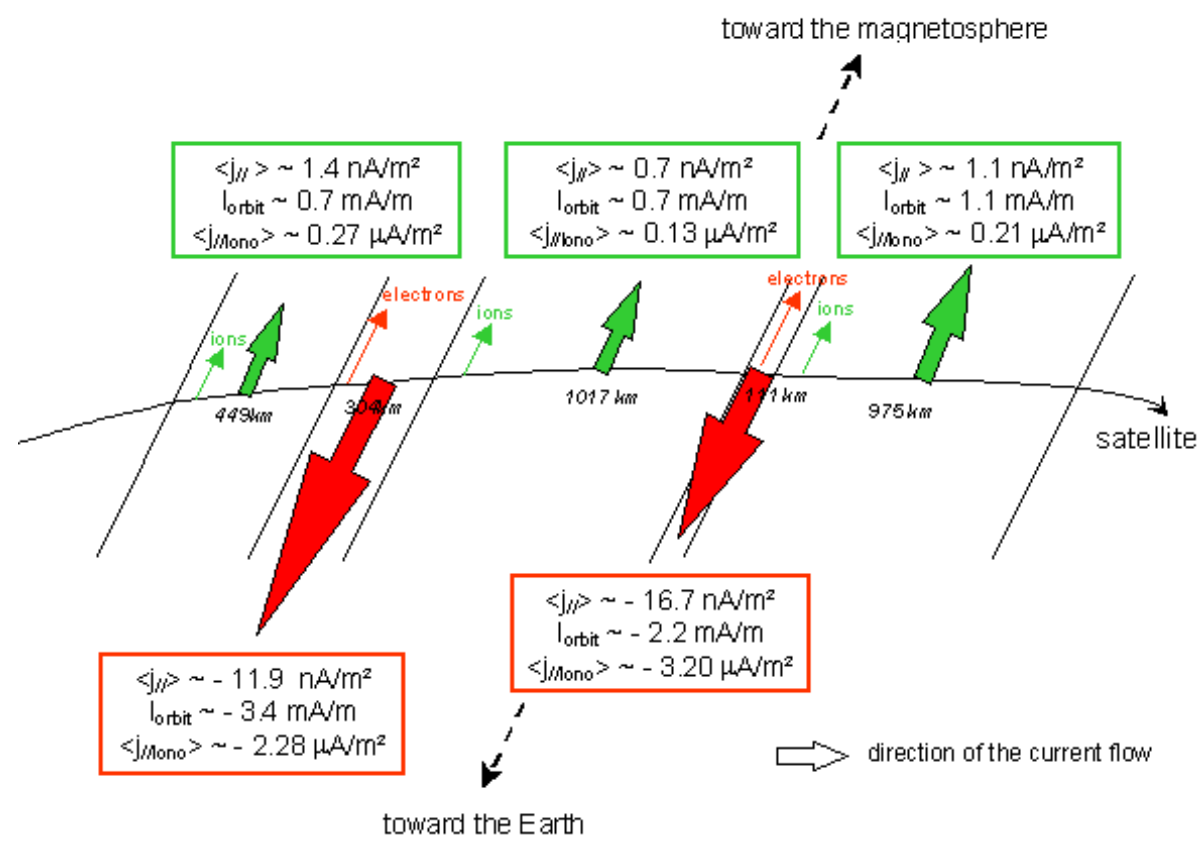

Fig. 13. Summary sketch of the suggested current geometry for a polar cap crossing by northward IMF. The red (green) arrows point toward the Earth (magnetosphere) and represent the downgoing (upgoing) currents mostly carried by the electron beams (ion structures). Encapsulated values correspond to the results obtained on 20 March 2003 between 00:38 and 00:51 UT.

ionospheric altitudes, as in the auroral zone (Carlson et al., 1998 b). They are also very variable on time scales shorter than one second.

These beams, detected just at the top of the photoelectron energy range, have energies of the order of $40-70 \mathrm{eV}$, occasionally reaching $100 \mathrm{eV}$. Their distribution functions are most of the time composed of a core, which can be satisfactorily approached with a Maxwellian, and of a suprathermal tail. The temperature estimated from the Maxwellian fit ranges typically between 2 and $20 \mathrm{eV}$, with a global trend of a heating increase with the energy gain. The heating suggests that these outflowing electrons have not only been accelerated but could also have experienced wave-particle interactions.

These beams interact with the much denser ambient plasma and meet the conditions to trigger a hot beam - hot plasma instability. This is confirmed by correlated observations from WHISPER of broadband electrostatic local emissions around the plasma frequency (a few $\mathrm{kHz}$ ).

At low altitude above the auroral zone, the acceleration of ionospheric electron beams is explained by the presence of a field-aligned potential drop (Carlson et al., 1998a, b). The test of a similar assumption for outflowing electron beams at high altitude above the polar cap requires favourable observation conditions: (i) energetic beams well separated from the photoelectron level and (ii) appropriate samplings of both particle and electric field measurements which imply observation time periods much longer than the spin period. Only a few events fulfil these conditions and therefore they cannot bring a formal evidence. However, their analysis argues in favour of the acceleration of ionospheric electrons by a fieldaligned potential drop, similarly to the auroral zone (Burch et al., 1983).

Generally, these upgoing electron beams are not isolated but surrounded by larger structures of field-aligned ion outflows. Indeed, both types of outflows follow one another along the spacecraft orbit. During northward or weak IMF conditions, the polar cap ionosphere actually appears as a wide region of upgoing particles, successively ions and electrons. This is illustrated by the sketch in Fig. 13 which, apart the amplitudes indicated for one specific polar cap crossing (on 20 March 2003), displays the features common to the studied events. The thin arrows show the successive outflows of the electrons (in red) inside narrow sheets and of the ions (in green) inside much wider sheets (Maggiolo et al., 2006). The thick arrows represent the current densities successively upward and downward. The upward current densities (green), of the order of $1 \mathrm{nA} / \mathrm{m}^{2}$, are mainly transported by the outflowing ions inside wide sheets while the outflowing electrons are the main carriers of downward current densities (red), of the order of $10 \mathrm{nA} / \mathrm{m}^{2}$, i.e., here, 10 times larger than the upward current densities. At ionospheric altitudes, it would produce current densities up to a few $\mu \mathrm{A} / \mathrm{m}^{2}$, comparable to auroral values (Burch et al., 1983). A rough estimate of the total current intensity inside each sheet leads to the conclusion that the current for a given polarity is balanced by the opposite polarity, within the uncertainties of the assumptions and measurements. 
Finally, Cluster observations at 5-9 $R_{E}$ altitudes reveal the polar ionosphere as a region of general electron and ion outflows during northward IMF conditions. The observation of these outflows, all along the orbit above the polar cap, points out the role of the polar ionosphere as a significant plasma source for the magnetosphere. These outflows participate to the closure of the current system distributed into successive sheets of opposite polarities which connect the polar ionosphere to distant regions of the magnetotail.

Acknowledgements. We are grateful to the EDI team and ESA Cluster Active Archive for providing electric field data.

Topical Editor I. A. Daglis thanks two referees for their help in evaluating this paper.

\section{References}

Andersson, L., Ergun, R. E., Newman, D. L., McFadden, J. P., Carlson, C. W., and Su, Y.-J.: Characteristics of parallel electric fields in the downward current region of the aurora, Phys. Plasma, 9, 3600-3609, 2002.

André, M. and Yau, A.: Theories and Observations of Ion Energization and Outflow in the High Latitude Magnetosphere, Space Sci. Rev., 80, 27-48, 1997.

Araki, T., Kamei, T., and Iyemori, T.: Polar cap vertical currents associated with northward interplanetary magnetic field, Geophys. Res. Lett., 11, 23-26, 1984

Balogh, A., Carr, C. M., Acuna, M. H., Dunlop, M. W., Beek, T. J., Brown, P., Fornaçon, K.-H., Georgescu, E., Glassmeier, K.H., Harris, J., Musmann, G., Oddy, T., and Schwingenschuh, K.: The Cluster Magnetic Field Investigation: overview of in-flight performance and initial results, Ann. Geophys., 19, 1207-1217, 2001 , http://www.ann-geophys.net/19/1207/2001/.

Boehm, M. H., Clemmons, J., Wahlund, J.-E., Eriksson, A., Eliasson, L., Blomberg, L., Kintner, P., and Höfner, H.: Observations of an upward-directed electron beam with the perpendicular temperature of the cold ionosphere, Geophys. Res. Lett., 22, 2103 2106, 1995.

Burch, J. L., Fields, S. A., and Heelis, R. A.: Polar cap electron acceleration regions, J. Geophys. Res., 84, 5863-5874, 1979.

Burch, J. L., Reiff, P. H., and Sugiura, M.: Upward electron beams measured by DE-1: a primary source of dayside region-1 Birkeland currents, Geophys. Res. Lett., 10, 753-756, 1983.

Carlson, C. W., Pfaff, R. F., and Watzin, J. G.: The Fast Auroral SnapshoT (FAST) mission, Geophys. Res. Lett., 25, 2013-2016, 1998a.

Carlson, C. W., McFadden, J. P., Ergun, R. E., Temerin, M., Peria, W., Mozer, F. S., Klumpar, D. M., Shelley, E. G., Peterson, W. K., Moebius, E., Elphic, R., Strangeway, R., Cattell, C., and Pfaff, R.: FAST observations in the downward auroral current region: Energetic upgoing electron beams, parallel potential drops, and ion heating, Geophys. Res. Lett., 25, 2017-2020, 1998b.

Cattell, C., Dombeck, J., Yusof, W., Carlson, C., and McFadden, J.: FAST observations of the solar illumination dependence of upflowing electron beams in the auroral zone, J. Geophys. Res., 109, A02209, doi:10.1029/2003JA010075, 2004.
Décréau, P. M. E., Fergeau, P., Krasnoselskikh, V., Le Guirriec, E., Lévêque, M., Martin, P., Randriamboarison, O., Rauch, J. L., Sené, F. X., Séran, H. C., Trotignon, J. G., Canu, P., Cornilleau, N., de Féraudy, H., Alleyne, H., Yearby, K., Mögensen, P. B., Gustafsson, G., André, M., Gurnett, D. C., Darrouzet, F., Lemaire, J., Harvey, C. C., Travnicek, P., and Whisper experimenters: Early results from the Whisper instrument on Cluster: an overview, Ann. Geophys., 19, 1241-1258, 2001, http://www.ann-geophys.net/19/1241/2001/.

Delcroix, J.-L. and Bers, A.: Physique des plasmas, edited by: CNRS Editions, 1994.

Elphic, R. C., Bonnell, J. W., Strangeway, R. J., Kepko, L., Ergun, R. E., McFadden, J. P., Carlson, C. W., Peria, W., Cattell, C. A., Klumpar, D., Shelley, E., Peterson, W., Moebius, E., Kistler, L., and Pfaff, R.: The auroral current circuit and field-aligned currents observed by FAST, Geophys. Res. Lett., 25, 2033-2036, 1998.

Ergun, R. E., Carlson, C. W., McFadden, J. P., Mozer, F. S., Delory, G. T., Peria, W., Chaston, C. C., Temerin, M., Elphic, R., Strangeway, R., Pfaff, R., Cattell, C. A., Klumpar, D., Shelley, E., Peterson, W., Meobius, E., and Kistler, L.: FAST satellite observations of electric field structures in the auroral zone, Geophys. Res. Lett., 25, 2025-2028, 1998.

Ergun, R. E., Andersson, L., Carlson, C. W., Newman, D. L., and Goldman, M. V.: Double layers in the downward current region of the aurora, Nonlin. Processes Geophys., 10, 45-52, 2003, http://www.nonlin-processes-geophys.net/10/45/2003/.

Evans, D. S.: Precipitating electron fluxes formed by a magnetic field aligned potential difference, J. Geophys. Res., 79, 28532858, 1974.

Fazakerley, A. N., Carter, P. J., Watson, G., Spencer, A., Sun, Y. Q., Coker, P., Kataria, D. O., Fontaine, D., Liu, Z. X., Gilbert, L., Lahiff, A. D., Mihaljcic, B., Szita, S., Taylor, M. G. G. T., Wilson, R. J., Dedieu, M., and Schwartz, S. J.: The Double Star Plasma Electron and Current Experiment, Ann. Geophys., 23, 2733-2756, 2005,

http://www.ann-geophys.net/23/2733/2005/.

Gustafsson, G., André, M., Carozzi, T., Eriksson, A. I., Fälthammar, C.-G., Grard, R., Holmgren, G., Holtet, J. A., Ivchenko, N., Karlsson, T., Khotyaintsev, Y., Klimov, S., Laakso, H., Lindqvist, P.-A., Lybekk, B., Marklund, G., Mozer, F., Mursula, K., Pedersen, A., Popielawska, B., Savin, S., Stasiewicz, K., Tanskanen, P., Vaivads, A., and Wahlund, J.-E.: First results of electric field and density observations by Cluster EFW based on initial months of operation, Ann. Geophys., 19, 1219-1240, 2001 , http://www.ann-geophys.net/19/1219/2001/.

Iijima, T. and Potemra, T. A.: The amplitude distribution of fieldaligned currents at northern high latitudes observed by Triad, J. Geophys. Res., 81, 2165-2174, 1976.

Iijima, T., Potemra, T. A., Zanetti, L. J., and Bythrow, P. F.: Large-scale Birkeland currents in the dayside polar region during strongly northward IMF: a new Birkeland current system, J. Geophys. Res., 89, 7441-7452, 1984.

Johnstone, A. D. and Winningham, J. D.: Satellite observations of suprathermal electron bursts, J. Geophys. Res., 87, 2321-2329, 1982.

Johnstone, A. D., Alsop, C., Burge, S., Carter, P. J., Coates, A J., Coker, A. J., Fazakerley, A. N., Grande, M., Gowen, R. A., 
Gurgiolo, C., Hancock, B. K., Narheim, B., Preece, A., Sheather, P. H., Winningham, J. D., and Woodliffe, R. D.: The CLUSTER and Phoenix Missions, PEACE: A Plasma Electron And Current Experiment, Space Sci. Rev., 79, 351-398, 1997.

Klumpar, D. M. and Heikkila, W. J.: Electrons in the ionospheric source cone: evidence for runaway electrons as carriers of downward Birkeland, Geophys. Res. Lett., 9, 873-876, 1982.

Maggiolo, R., Sauvaud, J.-A., Fontaine, D., Teste, A., Grigorenko, E., Balogh, A., Fazakerley, A., Paschmann, G., Delcourt, D., and Rème, H.: A multi-satellite study of accelerated ionospheric ion beams above the polar cap, Ann. Geophys., 24, 1665-1684, 2006, http://www.ann-geophys.net/24/1665/2006/.

Marklund, G., Blomberg, L., Fälthammar, C.-G., and Lindqvist, P.A.: On intense diverging electric fields associated with black aurora, Geophys. Res. Lett., 21, 1859-1862, 1994.

McFadden, J. P., Carlson, C. W., and Ergun, R. E.: Microstructure of the auroral acceleration region as observed by FAST, J. Geophys. Res., 104, 14 453-14 480, 1999.

Miyake, W., Mukai, T., and Kaya, N.: A statistical study of fieldaligned electron beams associated with ion conics events, Ann. Geophys., 16, 940-947, 1998, http://www.ann-geophys.net/16/940/1998/.

Mozer, F. S. and Kletzing, C. A.: Direct observation of large, quasistatic, parallel electric fields in the auroral acceleration region, Geophys. Res. Lett., 25, 1629-1632, 1998.

Paschmann, G., Quinn, J. M., Torbert, R. B., Vaith, H., McIlwain, C. E., Haerendel, G., Bauer, O. H., Bauer, T., Baumjohann, W., Fillius, W., Förster, M., Frey, S., Georgescu, E., Kerr, S. S., Kletzing, C. A., Matsui, H., Puhl-Quinn, P., and Whipple, E. C.: The Electron Drift Instrument on Cluster: overview of first results, Ann. Geophys., 19, 1273-1288, 2001, http://www.ann-geophys.net/19/1273/2001/.

Rème, H., Aoustin, C., Bosqued, J.-M., Dandouras, I., Lavraud, B., Sauvaud, J.-A., Barthe, A., Bouyssou, J., Camus, T., Coeur-Joly, O., Cros, A., Cuvilo, J., Ducay, F., Garbarowitz, Y., Medale, J.L., Penou, E., Perrier, H., Romefort, D., Rouzaud, J., Vallat, C., Alcaydé, D., Jacquey, C., Mazelle, C., D’Uston, C., Möbius, E., Kistler, L. M., Crocker, K., Granoff, M., Mouikis, C., Popecki, M., Vosbury, M., Klecker, B., Hovestadt, D., Kucharek, H., Kuenneth, E., Paschmann, G., Scholer, M., Sckopke, N., Seidenschwang, E., Carlson, C. W., Curtis, D. W., Ingraham, C., Lin, R. P., McFadden, J. P., Parks, G. K., Phan, T., Formisano, V., Amata, E., Bavassano-Cattaneo, M. B., Baldetti, P., Bruno, R., Chionchio, G., di Lellis, A., Marcucci, M. F., Pallocchia, G., Korth, A., Daly, P. W., Graeve, B., Rosenbauer, H., Vasyliunas, V., McCarthy, M., Wilber, M., Eliasson, L., Lundin, R., Olsen, S., Shelley, E. G., Fuselier, S., Ghielmetti, A. G., Lennartsson, W., Escoubet, C. P., Balsiger, H., Friedel, R., Cao, J.-B., Kovrazhkin, R. A., Papamastorakis, I., Pellat, R., Scudder, J., and Sonnerup, B.: First multispacecraft ion measurements in and near the Earth's magnetosphere with the identical Cluster ion spectrometry (CIS) experiment, Ann. Geophys., 19, 1303-1354, 2001,

http://www.ann-geophys.net/19/1303/2001/.
Shelley, E. G.: Erratum: Correction "The polar ionosphere as a source of energetic magnetospheric plasma", Geophys. Res. Lett., 9, 941-944, 1982, Geophys. Res. Lett., 9, 1298-1298, 1982.

Shinohara, I. and Kokubun, S.: Statistical properties of particle precipitation in the polar cap during intervals of northward interplanetary magnetic field, J. Geophys. Res., 101, 69-82, 1996.

Szita, S., Fazakerley, A. N., Carter, P. J., James, A. M., Travnicek, P., Watson, G., André, M., Eriksson, A., and Torkar, K.: Cluster PEACE observations of electrons of spacecraft origin, Ann. Geophys., 19, 1721-1730, 2001, http://www.ann-geophys.net/19/1721/2001/.

Temerin, M. and Carlson, C. W.: Current-Voltage relationship in the downward auroral current region, Geophys. Res. Lett., 25, 2365-2368, 1998.

Torkar, K., Riedler, W., Escoubet, C. P., Fehringer, M., Schmidt, R., Grard, R. J. L., Arends, H., Rüdenauer, F., Steiger, W., Narheim, B. T., Svenes, K., Torbert, R., André, M., Fazakerley, A., Goldstein, R., Olsen, R. C., Pedersen, A., Whipple, E., and Zhao, H.: Active spacecraft potential control for Cluster - implementation and first results, Ann. Geophys., 19, 1289-1302, 2001, http://www.ann-geophys.net/19/1289/2001/.

Tsyganenko, N. A.: Global quantitative models of the geomagnetic field in the cislunar magnetosphere for different disturbance levels, Planet. Space Sci., 35, 1347-1358, 1987.

Yau, A. W. and André, M.: Sources of Ion Outflow in the High Latitude Ionosphere, Space Sci. Rev., 80, 1-25, 1997.

Zhu, L., Schunk, R. W., and Sojka, J. J.: Polar cap arcs: a review, J. atmos. terr. Phys. 59, 1087-1126, 1997. 